\title{
Modified Inverse Source Coding for Diffusion Based Molecular Communication System
}

\author{
Balaji Dhayabaran*, G. Thavasi Raja*, and Maurizio Magarini! \\ *Department of Electronics and Communication Engineering \\ National Institute of Technology,Trichy, India,Email: balajidhayabaran@gmail.com,thavasi@nitt.edu \\ 'Dipartimento di Elettronica, Informazione e Bioingegneria \\ Politecnico diMilano, Milano, Italy, Email: maurizio.magarini@polimi.it
}

\begin{abstract}
In this paper we prove that Inter-Symbol Interference (ISI) and Bit Error Rate (BER) of a Diffusion Based Molecular Communication (DBMC) system depend on the apriori probability of the transmitted data by deriving lower and upper bounds for the number of received molecules. We also compute the optimal value of a-priori probability for which ISI and BER are minimized. A source coding technique, called Modified Inverse Source Coding (MISC), is proposed that allows to control the a-priori probability of the transmitted data. The results show that the MISC-DBMC system not only provides a better BER performance compared to an uncoded one but also improves the range of communication.
\end{abstract}

Index Terms-Diffusive channel, molecular communication, inverse source coding, inter-symbol interference, bit error rate.

\section{INTRODUCTION}

$\mathbf{E}$ NGINEERED biological nanomachines can be used for applications like health care and genetic engineering, which requires robust and reliable communication between them. A biologically inspired communication technology, called molecular communication (MC), can be used to establish such a communication by encoding information into messenger molecules, which act as information carriers [1]. The information can be encoded into the number of molecules released (Concentration Shift Keying) [2], [3], or the type of molecules (Molecular Shift Keying) [4], or the release time of the molecules (Timing modulation) [5], etc.

One of the major challenges in Diffusion Based Molecular Communication (DBMC) is Inter-Symbol Interference (ISI), which arises due to the propagation delay caused by the diffusion of molecules from the transmitting to the receiving node. A method to mitigate the effect of ISI, reported in [6], uses active enzymes that freely diffuse in the propagation environment. The enzymes react with the information molecules and form reaction intermediate and then degrade them so that they do not interfere with future transmissions. A new modulation technique called Molecular Transition Shift Keying (MTSK) along with a low complex decision feedback equalizer was proposed in [7] to increase the data rate by suppressing the ISI. An optimal filter was designed in [8] to maximize the signal to interference plus noise ratio.

Another popular method to counter ISI is by using error correction coding schemes. The performance of various error control coding techniques have been compared for different scenarios for DBMC in [9]. An energy efficient coding technique for MC was introduced in [10]. An ISI-aware channel coding technique that incorporates the effect of ISI in channel code design for DBMC channel was proposed in [11].

In this paper, a new coding technique to reduce the effect of ISI in DBMC is proposed. This new coding scheme is inspired by Inverse Source Coding (ISC) technique originally proposed to control the dimming percentage in visible light communication [12]. We evaluate the Bit Error Rate (BER) and perform capacity analysis of the proposed scheme. The most significant contributions of this paper are the following:

1) Derivation of lower and upper bounds for the number of received molecules at the end node. Using the derived bounds we prove that the effect of ISI can be minimized by reducing the percentage of 1's in the transmit data.

2) Proposal of a Modified ISC (MISC) scheme for DBMC system to control the percentage of 1's and 0's in the transmitted data. Capacity and BER performance of MISC-DBMC system are compared with that of the uncoded DBMC system.

This paper is organized as follows. The model of the DBMC system is explained in Sec. II. The upper and lower bound on the number of molecules at the receiver is derived in Sec. III and IV, respectively. The MISC scheme is introduced in Sec. V. Numerical results are presented and discussed in Sec. VI. Finally, the paper is concluded in Sec. VII.

\section{SySTEM MODEL}

A three dimensional DBMC system with a fully absorbing spherical receiver of radius $R \mu \mathrm{m}$ separated by a distance $r \mu \mathrm{m}$ from a point transmitter is considered, as illustrated in Fig. 1. The information bits are transmitted sequentially using On-Off Keying (OOK) modulation, where bit 1 is represented by releasing $N_{t x}$ number of molecules at the beginning of the symbol duration $\left(t_{s}\right)$ and bit 0 is represented by releasing no molecules from the transmitter. At the receiver the information is decoded by measuring the concentration of received molecules distorted by ISI, diffusion noise, counting noise, and environment noise for each symbol duration $\left(t_{s}\right)$. The memory of the MC channel, or the length of ISI, is assumed to be $I$. The molecular concentration $\left(N_{c}\right)$ measured at the receiver during the current time slot $c$ is [13] 


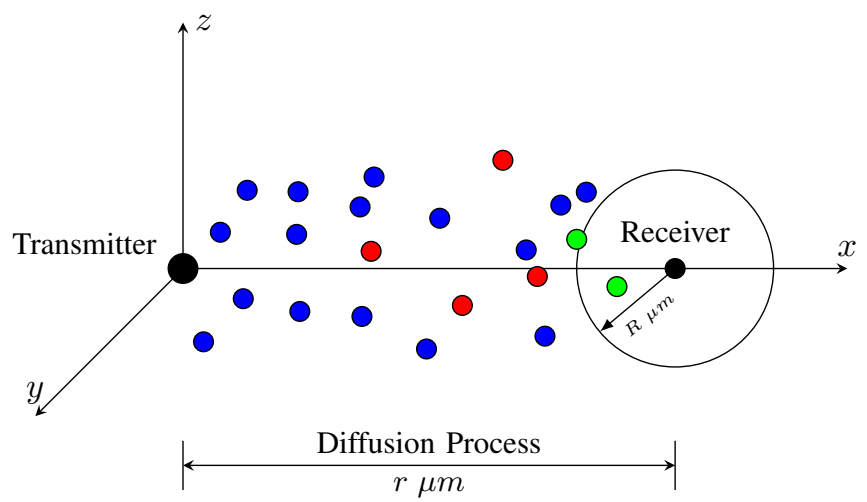

Fig. 1. Model of the considered DBMC system: molecules emitted by the transmitter are shown in blue, interfering molecules are shown in red, and absorbed molecules are shown in green.

$$
N_{c}=b_{c} N_{0}+\sum_{i=1}^{I} b_{c-i} N_{i}+W_{c}^{c n t}+W_{c}^{i n t},
$$

where $b_{c}$ is the information bit transmitted in the current time slot, $b_{c-i}$ is the information bit transmitted $i$ time slots before the current one, $b_{c-i}, b_{c} \in\{0,1\}, i=1,2,3, \cdots, I$, being $I$ the length of ISI, $N_{i}$ is a random variable that denotes the number of molecules received at the receiver in the $i$ th time slot, out of $N_{t x}$ molecules released from the transmitter $i$ time slots before. $N_{i}$ can be modeled as a Gaussian distributed random variable assuming that the expected number of received information molecules is large [14]:

$$
N_{i} \sim \mathcal{N}\left(N_{t x}\left(P_{i+1}-P_{i}\right), N_{t x}\left(P_{i+1}-P_{i}\right)\left(1-P_{i+1}+P_{i}\right)\right),
$$

where $P_{i}$ is the capture probability of the molecule at the receiver until time $\left(i \times t_{s}\right)$ after it has been released from the transmitter $i$ time slots before. This probability, which was derived in [9], [15], is given by

$$
P_{i}=P_{h i t}\left(r, i \times t_{s}\right)=P_{i}=\frac{R}{r} \operatorname{erfc}\left(\frac{(r-R)}{2 \sqrt{D\left(i \times t_{s}\right)}}\right),
$$

where $\operatorname{erfc}(\cdot)$ is the complementary error function [16]. In addition to ISI, the concentration of molecules measured in the current time slot at the receiver is also affected by the counting noise and by the interfering molecules in the environment or those produced by the other transmitting nodes that are represented as $W_{c}^{c n t}$ and $W_{c}^{i n t}$, respectively. Both noise components can be modeled as Gaussian distributed random variables assuming that the expected number of interfering noise molecules is large i.e. $W_{c}^{\text {cnt }} \sim \mathcal{N}\left(0, \sigma_{\text {cnt }}^{2}\right)$ and $W_{c}^{\text {int }} \sim$ $\mathcal{N}\left(0, \sigma_{\text {int }}^{2}\right)$, respectively [14]. The counting noise is a signal dependent noise with variance $\sigma_{c n t}^{2}=\left(\sum_{i=0}^{I} b_{c-i} N_{i}\right) / V_{R}$, where $V_{R}$ is the volume of the receiver [3]. Assuming that the expected number of received information and noise molecules is large, the total number of molecules received in the current time slot $\left(N_{c}\right)$ can be approximated as [14]:

$$
N_{c} \sim \mathcal{N}\left(\mu_{c}, \sigma_{c}^{2}\right) \text {, }
$$

\begin{tabular}{|c|c|c|c|c|c|c|}
\hline$b_{c}$ & 0 & 1 & 1 & - & - $\bullet$ & 1 \\
\hline Capture $\longrightarrow$ & 0 & $\hat{P}_{2}$ & $\hat{P}_{3}$ & - & • & $\hat{P}_{I}$ \\
\hline Lower bound $\longrightarrow$ & 0 & $\hat{P}_{b 2}=\hat{P}_{2}$ & 0 & $\bullet$ & - & 0 \\
\hline
\end{tabular}

where $\mu_{c}$ is the expected number of molecules received in the current time slot given as, $\mu_{c}=\sum_{i=0}^{I} b_{c-i} N_{i}=b_{c} N_{t x} P_{1}+$ $\sum_{i=1}^{I} b_{c-i} N_{t x}\left(P_{i+1}-P_{i}\right)$. The second term on the right
Fig. 2. Example of a Transmit bit sequence and the corresponding capture probabilities $\left(\hat{P}_{i}\right)$, and lower bound approximation. $b_{c}$ is the information bit in the current time slot, and other symbols contribute to ISI.

hand side of the equation is due to ISI, and is given by, $\sigma_{c}^{2}=$ $\sum_{i=0}^{I} b_{c-i} N_{t x}\left(P_{i+1}-P_{i}\right)\left(1-P_{i+1}+P_{i}\right)+\sigma_{c n t}^{2}+\sigma_{i n t}^{2}$.

\section{LOWER BOUND ON THE NUMBER OF RECEIVED MOLECULES}

In this section a lower bound is derived for the number of molecules received at the receiver node during the current time slot. Let us define the vector $\hat{P}=$ $\left[\left(P_{2}-P_{1}\right),\left(P_{3}-P_{2}\right), \cdots,\left(P_{I+1}-P_{I}\right)\right]$, whose elements are ordered in non-increasing way as

$$
\hat{P}=\left\{\left(\hat{P}_{1}, \hat{P}_{2}, \hat{P}_{3}, \cdots \hat{P}_{I}\right) \in \mathcal{R}^{I} \mid \hat{P}_{1}>\hat{P}_{2}>\hat{P}_{3} \cdots \hat{P}_{I}\right\},
$$

where $\hat{P}_{i}=\left(P_{i+1}-P_{i}\right), i \in\{1,2,3, \cdots, I\}$ denotes the capture probability of ISI molecules at the receiver that are released $i$ time slots before the current one from the transmitter as shown in Fig. 2. The impulse response of the DBMC channel reaches its maximum at a time $t_{\max }$ and then decreases monotonically [3]. By choosing the symbol duration $T_{s}$ such that $T_{s} \gg t_{\max }$, we can ensure that $\hat{P}_{i}$ decreases as $i$ increases from 1 to $I$. The expression of the lower bound for the number of molecules received in the current time slot is

$$
N_{c}^{L}=b_{c} N_{t x} P_{1}+N_{t x} \hat{P}_{b_{i}}+\bar{W}_{c} \leqslant N_{c},
$$

where $\bar{W}_{c}=W_{c}^{c n t}+W_{c}^{i n t}$, which takes into account the combined noise components, is distributed as $\bar{W}_{c} \sim \mathcal{N}\left(0, \sigma_{w}^{2}\right)$, $\sigma_{w}^{2}=\sigma_{c n t}^{2}+\sigma_{i n t}^{2}$ is the combined noise variance, and $\hat{P}_{b_{i}}$ is equal to $\hat{P}_{i}$ corresponding to the first event of $b_{i}=1$, as illustrated in Fig. 2. The lower bound approximation of the received number of molecules in the current symbol slot follows a Gaussian distribution

$$
\begin{aligned}
N_{c}^{L} \sim \mathcal{N} & \left(b_{c} N_{t x} P_{1}+N_{t x} \hat{P}_{b_{i}},\right. \\
& \left.b_{c} N_{t x} P_{1}\left(1-P_{1}\right)+N_{t x} \hat{P}_{b_{i}}\left(1-\hat{P}_{b_{i}}\right)+\sigma_{w}^{2}\right) .
\end{aligned}
$$

From the expression of the lower bound given in (6), which is illustrated in Fig. 2, it is clear that in the lower bound approximation only one bit in the ISI symbols will take a value equal to 1 , i.e., $b_{j}=0, \forall j \neq i$.

Theorem 1: Let the random variable $b \in\{0,1\}$ have a Probability Mass Function (PMF) defined by $P(1)=p, P(0)=$ $q=1-p$. If $b_{1}, b_{2}, b_{3}, \cdots, b_{I}$ are Independent and Identically Distributed (i.i.d) with $P(b)$ then the value of $p$ that minimizes the ISI in (1) is given by $p=1 / I, I$ being the length of ISI.

Proof: Let the random variable $X \in\{1,2,3, \ldots, I\}$ represent the position of the bit in the ISI symbols. The symbols emitted by the source $b_{1}, b_{2}, \cdots, b_{I}$ are assumed to be i.i.d. Let us define a random experiment of repeated 
independent Bernoulli trials until a success is obtained, with probability of success at each independent trial equal to $p$. Then $X$ is considered as a geometric random variable. The event that the bit $b_{i}$ takes a value of 1 is equivalent to getting the success in the $i$ th trial of the random experiment. The probability of that event is given by

$$
P\left(b_{i}=1\right)=P(X=i)=q^{i-1} p .
$$

The mean position at which the first success occurs is given by $E(X)=1 / p$. The effect of ISI over the current symbol is minimum only if the last bit of ISI symbol takes a value of 1 i.e., $b_{I}=1$, and $b_{i}=0, \forall i \neq I$. The probability of this event is

$$
P\left(b_{I}=1\right)=P(X=I)=q^{I-1} p .
$$

The effect of ISI can be minimized by maximizing (9) as

$$
\underset{p}{\operatorname{argmax}}\left\{P\left(b_{I}=1\right)\right\}=\underset{p}{\operatorname{argmax}}\left(q^{I-1} p\right) .
$$

Taking the logarithm on both sides of (10) will result in

$$
\underset{p}{\operatorname{argmax}} \log \left\{P\left(b_{I}=1\right)\right\}=\underset{p}{\operatorname{argmax}}\{(I-1) \log q+\log p\} .
$$

The value of $p$ that maximizes (11) is determined by differentiating (11) and equating it to zero. It is found that its value is $p=1 / I$. This completes the proof.

The total average probability of error for the DBMC system using a threshold detector at the receiver is [13]

$$
P_{e}=\sum_{j=1}^{2^{I}}\left(P_{01, j}+P_{10, j}\right),
$$

where

$P_{01, j}=q^{i} p \mathbb{Q}\left(\frac{\mu_{01, j}-\tau}{\sigma_{01, j}}\right), P_{10, j}=q^{i-1} p^{2} \mathbb{Q}\left(\frac{\tau-\mu_{10, j}}{\sigma_{10, j}}\right)$,

$\mu_{01, j}=N_{t x} \hat{P}_{i j}, \quad \mu_{10, j}=N_{t x} P_{1}+N_{t x} \hat{P}_{i j}$,

$\sigma_{01, j}=\sqrt{N_{t x} \hat{P}_{i j}\left(1-\hat{P}_{i j}\right)+\sigma_{w}^{2}}$, and

$\sigma_{10, j}=\sqrt{N_{t x} P_{1}\left(1-P_{1}\right)+N_{t x} \hat{P}_{i j}\left(1-\hat{P}_{i j}\right)+\sigma_{w}^{2}}$

' $j$ ' being the bit sequence index, $P_{m n, j}$ is the event of erroneously decoding bit ' $m$ ' as ' $n$ ' when the ISI is $j$ th bit sequence, $(m, n) \in\{0,1\}, \hat{P}_{i, j}$ is $\hat{P}_{b_{i}}$ corresponding to the $j$ th bit sequence, $\tau$ is the threshold of the detector at the receiver, and $\mathbb{Q}$ is the cumulative standard Gaussian distribution function. Thus, it can be concluded that the BER in the lower bound scenario depends on the position of the first occurrence of bit 1 in the ISI bit sequence.

Remark 1: The ISI can be made zero by choosing $p$ very close to zero, but that would mean that there is no information transfer. Thus the value of $p$ should be chosen such that the target average rate of transmission is achieved.

\section{UPPER BOUND ON THE NUMber OF RECEIVED MOLECULES}

The upper bound on the number of received molecules in the current symbol slot is given by

$$
N_{c}^{U}=b_{c} N_{t x} P_{1}+N_{t x} \sqrt{K} \hat{P}_{b_{i}}+\bar{W}_{c} \geqslant N_{c},
$$

where $K=(I \times p)$ denotes the average number of one's in the binary sequence with apriori probability of $p$. The number of molecules received in the current time slot in the upper bound scenario follows a Gaussian distribution

$$
\begin{gathered}
N_{c}^{U} \sim \mathcal{N}\left(b_{c} N_{t x} P_{1}+\sqrt{K} N_{t x} \hat{P}_{b_{i}},\right. \\
\left.b_{c} N_{t x} P_{1}\left(1-P_{1}\right)+K N_{t x}\left(\hat{P}_{b_{i}}\right)\left(1-\hat{P}_{b_{i}}\right)+\sigma_{w}^{2}\right) .
\end{gathered}
$$

Theorem 2: If the number of received molecules is given by $N_{c}^{U}<b_{c} N P_{1}+N \sqrt{K} \hat{P}_{b_{i}}$, then the value of $p$ that minimizes the magnitude of ISI is given by $p=1 /\{I+\log (q)\}$.

Proof: The proof is similar to that illustrated for theorem 1 in Sec. III. Moreover, it is important to consider that according to Asymptotic Equi-partition Property (AEP), the typical binary sequence in the ISI symbols will contain $K$ number of 1's [17]. From this understanding it is straightforward to show that when there are $K 1$ 's in the ISI symbols, minimum ISI occurs when the first 1 occurs at bit $b_{I-K}$. The probability of this event is given by

$$
P\left(b_{I-K}=1\right)=q^{I-K-1} p .
$$

To reduce the effect of ISI this probability should be maximized as

$$
\underset{p}{\operatorname{argmax}}\left\{P\left(b_{I-K}=1\right)\right\}=\underset{p}{\operatorname{argmax}}\left\{q^{I-K-1} p\right\} .
$$

Taking the logarithm on both sides of (16) will result in

$$
\begin{aligned}
\underset{p}{\operatorname{argmax}} & \left\{\log \left(P\left(b_{I-K}=1\right)\right)\right\} \\
& =\underset{p}{\operatorname{argmax}}\{(I-K-1) \log (1-p)+\log (p)\} .
\end{aligned}
$$

Differentiating (17) with respect to $p$ and equating to zero

$$
(I-I p-1) \times \frac{1}{(1-p)} \times(-1)+(-I) \times \log (1-p)+\frac{1}{p}=0 .
$$

From (18), we can find that, (15) is maximized by ' $p=1 /\{I+$ $\log (q)\}^{\prime}$, which completes the proof.

The probability of error for the upper bound scenario is calculated using (12) with the following parameters

$$
\begin{aligned}
\mu_{01, j} & =\sqrt{K} N_{t x} \hat{P}_{i j}, \mu_{10, j}=N_{t x} P_{1}+\sqrt{K} N_{t x} \hat{P}_{i j}, \\
\sigma_{01, j} & =\sqrt{K N_{t x}\left(\hat{P}_{i j}\right)\left(1-\hat{P}_{i j}\right)+\sigma_{w}^{2}}, \\
\sigma_{10, j} & =\sqrt{N_{t x} P_{1}\left(1-P_{1}\right)+K N_{t x}\left(\hat{P}_{i j}\right)\left(1-\hat{P}_{i j}\right)+\sigma_{w}^{2}} .
\end{aligned}
$$

From (19), it is clear that the BER for the upper bound scenario not only depend on the position of bit 1 in the ISI bit sequence but also depend on the number of 1's in the ISI symbol. 
TABLE I

HUFFMAN CODING FOR $p=0.2$.

\begin{tabular}{|c|c|c|}
\hline Symbol/Length & Probability & Codeword/Length \\
\hline $00 / 2$ & 0.64 & $1 / 1$ \\
\hline $01 / 2$ & 0.16 & $00 / 2$ \\
\hline $10 / 2$ & 0.16 & $011 / 3$ \\
\hline $11 / 2$ & 0.04 & $010 / 3$ \\
\hline
\end{tabular}

TABLE II

INVERSE HUFFMAN CODING FOR $p=0.2$.

\begin{tabular}{|c|c|c|}
\hline Symbol/Length & Probability & Codeword/Length \\
\hline $010 / 3$ & 0.125 & $1 / 1$ \\
\hline $011 / 3$ & 0.125 & $01 / 2$ \\
\hline $00 / 2$ & 0.25 & $001 / 3$ \\
\hline $1 / 1$ & 0.5 & $000 / 3$ \\
\hline
\end{tabular}

\section{Modified Inverse Source Coding For DBMC}

In this section the MISC technique is introduced, which can be used to control the percentage of 1's and 0's in the transmitted data. In conventional source coding techniques, the highly probable information symbols are assigned codewords with shorter length and the less probable ones are assigned codewords with longer length, thus reducing the average codeword length and resulting in better spectral efficiency [17]. In MISC, the highly probable information symbols are assigned codewords with higher length and smaller codeword weight and vice versa. The MISC results in reduction of ISI, and better BER performance in DBMC systems with OOK modulation. But a major disadvantage of this technique is that MISC results in decompression of transmit data that leads to reduction in the capacity of the DBMC system.

In this paper Huffman coding is used to construct Inverse Huffman codes. Let us assume that the desired percentage of 1 's and 0 's should be $20 \%$ and $80 \%$ respectively. Huffman encoding is used to generate codewords with optimal length. These codewords are then used to construct inverse Huffman codes by assigning largest length code to symbols with high probability of occurrence and smallest length codes to symbols with less probability of occurrence. In MISC the highly probable symbols are assigned 0's instead of 1's as is the normal case in Huffman coding. Huffman code and corresponding inverse Huffman code for $p=0.2$ and $q=0.8$ are shown in Tables I and II. MISC achieves the desired target percentage of 1's in the codeword

$$
\frac{1 \times 0.125+1 \times 0.125+1 \times 0.25}{3 \times 0.5+2 \times 0.25+1 \times 0.125} \approx 0.235(\simeq 20 \%),
$$

where the numerator is the average number of 1's in the codeword and the denominator is the average codeword length.

\section{Results AND Discussions}

In this section, we first present the performance of DBMC system model shown in Fig. 1, considering the lower and upper bound scenarios. Then, the performance of the MISC-DBMC system for a simple threshold detector is analyzed. In all the cases, BER is considered as the performance metric. To show that the MISC technique improves the communication range
TABLE III

SiMULATION PARAMETERS

\begin{tabular}{|l|c|c|}
\hline \multicolumn{1}{|c|}{ Description } & Variable & Value \\
\hline Diffusion coefficient of the medium & $D$ & $79.4 \mu \mathrm{m}^{2} / \mathrm{s}$ \\
\hline Radius of the spherical receiver & $R$ & $1 \mu \mathrm{m}$ \\
\hline Distance between transmitter and receiver & $r$ & $4 \mu \mathrm{m}, 6 \mu \mathrm{m}, 8 \mu \mathrm{m}$ \\
\hline Symbol duration & $T_{s}$ & $4 \times t_{\max }=4 \times \frac{r^{2}}{6 D}$ \\
\hline Channel memory / ISI length & $I$ & 10 \\
\hline
\end{tabular}

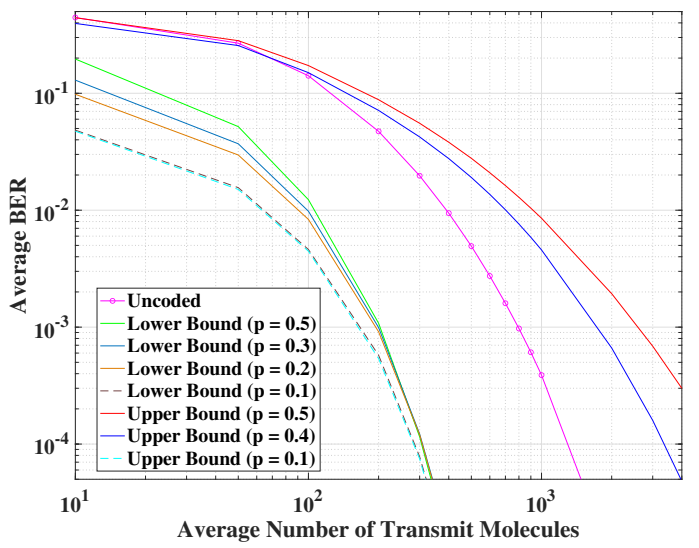

Fig. 3. BER performance lower and upper bound approximations compared with uncoded DBMC for different values of ' $p$ ' and $r=4 \mu \mathrm{m}$.

of DBMC, a performance comparison of coded and uncoded systems is done for multiple values of $r$. The simulation parameters used in this paper are given in Table III. Normalization of symbol duration $t_{s}$ and average number of molecules $N_{t x}$ to emit for bit 1 for a coded system is performed for a fair comparison between coded and uncoded systems [11].

The BER of uncoded DBMC system is compared with that produced by the lower and upper bounds in Fig. 3. The numerical results clearly show the performance improvement in both upper and lower bounds as the value of a-priori probability $p$ decreases. It is also shown that the lowest BER for the lower bound performance is achieved when $p=0.1$, i.e., $p=1 / I$, and the best performance in the upper bound scenario is achieved when $p=1 /\{I+\log (q)\}$. The performance of the upper bound matches with the lower bound when $q=0.9 \Longrightarrow p=0.1$ as expected.

The performance comparison of the MISC-DBMC system using a threshold detector for different values of $p$ and $r$ is shown in Fig. 4. This figure shows that, the BER performance of the MISC-DBMC system improves as the value of $p$ reduces. Coding gain as defined in [9] can be used to measure the performance improvement of the MISC coding scheme. At $\mathrm{BER}=10^{-2}$ the coding gain of MISC-DBMC system with $p=0.2$ and $r=4 \mu \mathrm{m}, 6 \mu \mathrm{m}, 8 \mu \mathrm{m}$ are shown to be $3 \mathrm{~dB}, 3.25 \mathrm{~dB}$, and $1.63 \mathrm{~dB}$, respectively. Similarly, for $p=0.3$ the coding gain are shown to be $1.24 \mathrm{~dB}, 1.6 \mathrm{~dB}$, and $0.9 \mathrm{~dB}$, respectively. As the range $r$ increases from $2 \mu \mathrm{m}$ to $4 \mu \mathrm{m}$ the coding gain increases slightly and reduces to almost half at $r=8 \mu \mathrm{m}$ in both cases of $p=0.2,0.3$. It is also worth 


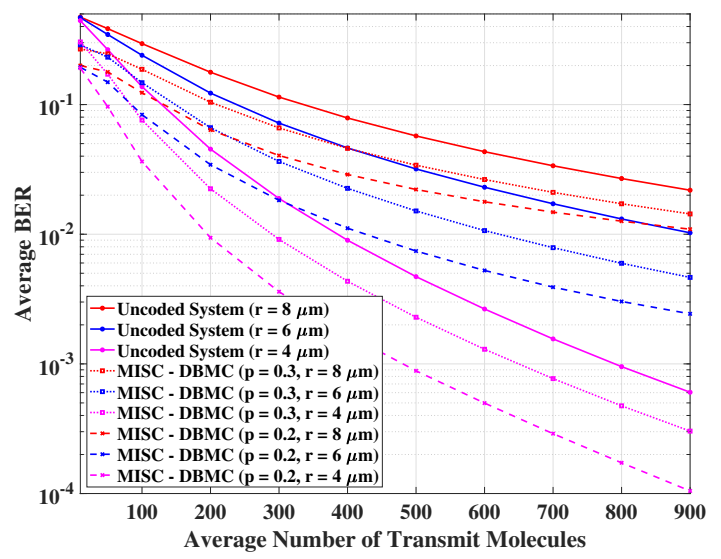

Fig. 4. BER performance comparison of DBMC, and MISC-DBMC for different values of ' $p$ '.

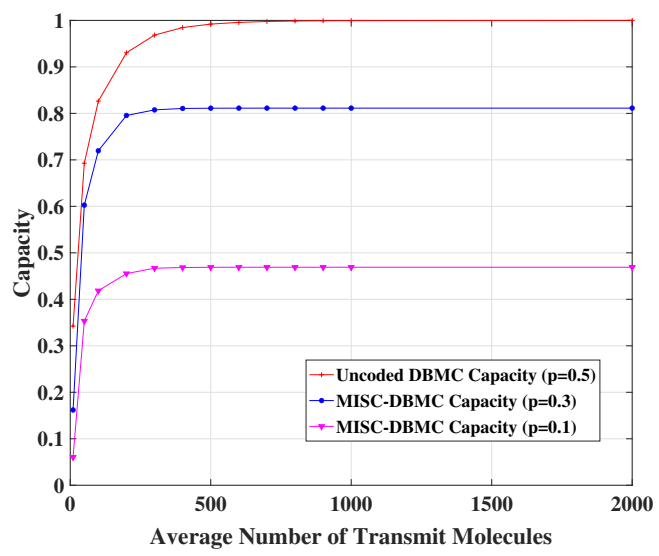

Fig. 5. Channel capacity comparisons between DBMC, and MISC-DBMC for different values of ' $p$ '.

noting that as $p$ decreases from 0.3 to 0.2 the coding gain almost doubles for all values of $r$. Clearly from Fig. 4, it can be observed that the MISC-DBMC system with larger range performs better than the uncoded system with a shorter range till certain value of $N_{t x}$. For example, MISC-DBMC system with $p=0.2$ and $r=8 \mu \mathrm{m}$ performs better than the uncoded DBMC system with range $r=6 \mu \mathrm{m}$ till $N_{t x}=900$ after that the performance degrades slightly.

The capacity of the MISC-DBMC system for different values of $p$ is shown in Fig. 5. MISC affects the data rate due to the decompression of coded data. The average length before and after inverse Huffman coding given in Table II is 1.75 and 2.625 , respectively, with decompression ratio of $2.625 / 1.75 \simeq 1.5$. This decompression directly affects the data rate of the MISC-DBMC system. The capacity of DBMC system is given in [13]. It is shown in Fig. 5 that, in comparison to an uncoded DBMC system, the capacity of the MISC-DBMC system is less and the reduction in capacity depends on the decompression ratio of the corresponding MISC scheme.

\section{CONCLUSION}

In this paper, we have derived lower and upper bound approximations for the number of molecules observed at the receiver. These bounds are used to prove that the BER performance of a DBMC system depends on the a-priori probability of transmitted data and on the length of ISI. It is also proved that the BER performance improves by reducing the percentage of 1's in the transmitted data. Through mathematical derivations and simulations, we proved that the best BER performance of the DBMC system occurs when the value of $p$ is inverse of the ISI length. MISC technique proposed in this paper assigns code words of longer length and a smaller weight to symbols with high probability and vice versa. The MISC technique reduces the effect of ISI and improves the BER performance of the communication link. It is also shown through simulation that the MISCDBMC systems with larger distance of separation between transmitting and receiving nanomachines in comparison to uncoded systems display a superior BER performance, thus providing a reliable communication over longer distance.

\section{REFERENCES}

[1] N. Varshney, A. Patel, W. Haselmayr, A. K. Jagannatham, P. K Varshney, and A. Nallanathan, "Impact of intermediate nanomachines in multiple cooperative nanomachine-assisted diffusion advection mobile molecular communication," IEEE Trans. Commun., vol. 67, pp. 48564871, July 2019.

[2] M. Pierobon and I. F. Akyildiz, "A physical end-to-end model for molecular communication in nanonetworks," IEEE J. Sel. Areas Commun., vol. 28, pp. 602-611, May 2010.

[3] D. Kilinc and O. B. Akan, "Receiver design for molecular communication," IEEE J. Sel. Areas Commun., vol. 31, pp. 705-714, Dec. 2013.

[4] N. R. Kim and C. B. Chae, "Novel modulation techniques using isomers as messenger molecules for nano communication networks via diffusion," IEEE J. Sel. Areas Commun., pp. 847-856, Dec. 2013.

[5] K. V. Srinivas, A. W. Eckford, and R. S. Adve, "Molecular communication in fluid media: The additive inverse gaussian noise channel," IEEE Trans. Inf. Theory, vol. 58, pp. 4678-4692, Jul. 2012.

[6] A. Noel, K. C. Cheung, and R. Schober, "Improving receiver performance of diffusive molecular communication with enzymes," IEEE Trans. Nanobiosci., vol. 13, pp. 31-43, Mar. 2014.

[7] B. Tepekule, A. E. Pusane, H. B. Yilmaz, C. Chae, and T. Tugcu, "Isi mitigation techniques in molecular communication," IEEE Trans. Mol. Biol. Multi-Scale Commun., vol. 1, pp. 202-216, June 2015.

[8] V. Jamali, A. Ahmadzadeh, and R. Schober, "On the design of matched filters for molecule counting receivers," IEEE Commun. Lett., vol. 21 , pp. 1711-1714, Aug. 2017.

[9] Y. Lu, M. D. Higgins, and M. S. Leeson, "Comparison of channel coding schemes for molecular communications systems," IEEE Trans. Commun., vol. 63, pp. 3991-4001, Nov. 2015.

[10] C. Bai, M. S. Leeson, and M. D. Higgins, "Minimum energy channel codes for molecular communications," Electronic Letters, vol. 50, pp. 1669-1671, Nov. 2014.

[11] A. O. Kislal, H. B. Yilmaz, A. E. Pusane, and T. Tugcu, "ISI-Aware channel code design for molecular communication via diffusion," IEEE Trans. Nanobiosci., vol. 18, pp. 205-213, Apr. 2019.

[12] J. K. Kwon, "Inverse source coding for dimming in visible light communications using nrz-ook on reliable links," IEEE Photon. Technol. Lett., vol. 22, pp. 1455-1457, Oct. 2010.

[13] Y. Lu, M. D. Higgins, A. Noel, M. S. Leeson, and Y. Chen, "The effect of two receivers on broadcast molecular communication systems," IEEE Trans. Nanobiosci., vol. 15, pp. 891-900, Dec. 2016.

[14] V. Jamali, A. Ahmadzadeh, W. Wicke, A. Noel, and R. Schober, "Channel modeling for diffusive molecular communication-a tutorial review," Proceedings of the IEEE, vol. 107, pp. 1256-1301, Jul. 2019.

[15] H. B. Yilmaz, A. C. Heren, T. Tugcu, and C. Chae, "Three-dimensional channel characteristics for molecular communications with an absorbing receiver," IEEE Commun. Lett., vol. 18, pp. 929-932, Jun. 2014.

[16] J. Proakis and M. Salehi, Digital Communications, ser. McGraw-Hill International Edition. McGraw-Hill, 2008.

[17] T. M. Cover and J. A. Thomas, Elements of Information Theory. New York, NY, USA: Wiley-Interscience, 2006. 